\title{
Can admission notes be improved by using preprinted assessment sheets?
}

\author{
H M Goodyear, B W Lloyd
}

\begin{abstract}
Inpatient medical notes often fail to record important details of patient history and findings on clinical examination. To overcome problems with content and legibility of notes we introduced preprinted notes for the admission of children to this hospital. The quality of the information recorded for 100 children whose admissions were clerked with the preprinted notes was compared with that recorded for 100 whose admissions were recorded with the traditional notes. All case notes were selected randomly and retrospectively from traditional notes written from April to October 1993 and from preprinted notes written from October 1993 to April 1994. The quality of information was assessed according to the presence or absence of 25 agreed core clinical details and the number of words per clerking. In admissions recorded with the preprinted notes the mean number of core clinical details present was significantly higher than those recorded with traditional notes $(24.0 v 17 \cdot 6, \mathrm{p}<0.00001)$. Admissions recorded with the preprinted notes were also significantly shorter (mean 144 words $v 184$ words, p < 0.0001). The authors conclude that information about children admitted to hospital is both more complete and more succinct when recorded using preprinted admission sheets.
\end{abstract}

(Quality in Health Care 1995;4:190-193)

Keywords: paediatric admissions, admission notes

\section{Introduction}

Junior doctors often fail to record important details of both a patient's history and findings of the clinical examination when writing inpatient medical notes in the traditional style. ${ }^{12}$ In an audit of medical inpatient notes Gabbay et al found review of systems, social history, drugs taken, and allergies to be consistently badly recorded. ${ }^{1}$ In October 1993 in an attempt to improve the quality of admission notes we introduced a set of preprinted notes for each child admitted to our hospital, either from the accident and emergency department or directly to the children's wards. We investigated the effect of this innovation on the completeness and the brevity of admission notes.

\section{Methods}

Junior doctors were closely involved with the design of the preprinted admission sheets. A set of sheets consists of 4 sides of coloured A4 paper preprinted with words and phrases prompting recording of core clinical details. In many sections of the sheets the doctor is required to ring the appropriate word or phrase and then write further details as necessary in the spaces provided. An example of a completed preprinted assessment form, giving fictional history and examination findings for an 18 month old girl who had a febrile convulsion is shown in the appendix.

Two hundred case notes of children admitted to this hospital were randomly selected (100 traditional notes written between April and October 1993 and 100 notes recorded on the preprinted sheets and written between October 1993 and April 1994). Each set of admission notes was assessed by one of us (HMG) with a proforma as a checklist for the presence or absence of the 25 core clinical details (box), previously agreed after considerable discussion in an audit meeting of quality of case notes. The number of words

\begin{tabular}{|rl|}
\hline \multicolumn{2}{|c|}{ Core clinical details } \\
1 & Name of child on every sheet \\
2 & Date \\
3 & Time \\
4 & Age \\
5 & Doctor's name at end of first entry \\
& (clearly legible) \\
6 & Presenting complaint(s) \\
7 & Child's wellbeing and activity in \\
& hours/days before admission \\
8 & How much the child has been \\
& eating/drinking before admission \\
9 & Drugs the child has been taking \\
10 & Birth weight \\
11 & Important perinatal problems \\
12 & Previous medical history \\
13 & Immunisation status \\
14 & Developmental history \\
15 & Details of health of siblings/parents \\
16 & Social history \\
17 & How ill the child is \\
18 & Temperature \\
19 & Perfusion \\
20 & Heart sounds and femoral pulses \\
21 & Signs of respiratory distress \\
22 & Chest clear or not \\
23 & Abdominal examination - masses \\
24 & Central nervous system - conscious level \\
25 & Problem list \\
\hline
\end{tabular}

\footnotetext{
Dr Lloyd

Accepted for publication 6 February 1995

Children's North Middlesex London N18 1Q roodyear, senior B W Lloyd, consultant paediatrician
} 
per clerking was counted for each set of notes. In the preprinted notes each item circled was counted as one word. The results were analysed by unpaired Student's $t$ tests.

\section{Results}

Of the 200 randomly selected case notes obtained from the medical records department, those on the preprinted sheets were significantly shorter and recorded significantly more core clinical details than the traditional notes (table).

The name of the child on every sheet of paper (detail 1) was the only core clinical detail not to show any change in frequency of recording between the preprinted sheets and the traditional notes. In particular, the notes written on the preprinted sheets were superior in the following ways: they were four times more likely to be signed legibly by the admitting doctor at the end of the first entry (detail 5), three times more likely to record the child's wellbeing and activity (detail 7), and three times more likely to record how well the child was perfused (detail 19). The following details were twice as likely to be recorded: how well the child was eating and drinking before admission (detail 8), drugs taken (detail 9), developmental history (detail 14), and social history (detail 16).

\section{Discussion}

Using preprinted admission sheets improved the quality of information recorded about children admitted to this hospital. These sheets contained significantly fewer words and therefore were presumably quicker both to write and to read. Over one year we found no disadvantages of preprinted assessment sheets compared with traditional style notes.

Both junior doctors and consultants in our department are enthusiastic about the new admission notes, which have become much neater and better organised. The structured nature of the preprinted notes helps to train junior doctors to write better notes, and in contrast to the variability in style of traditional admission notes, where to find particular details - for example, family history. Senior

Mean numbers ( $95 \%$ confidence interval) of core clinical details recorded and words per clerking in 200 sets of notes for paediatric inpatients

\begin{tabular}{lccc}
\hline Outcome & $\begin{array}{c}\text { Traditional notes } \\
(n=100)\end{array}$ & $\begin{array}{c}\text { Preprinted notes } \\
(n=100)\end{array}$ & p Value \\
\hline Core clinical details & $17 \cdot 6(17 \cdot 0$ to $18 \cdot 1)$ & $24(23 \cdot 7$ to $24 \cdot 2)$ & $<0 \cdot 00001$ \\
Words per clerking & $184(176$ to 192$)$ & $144(138$ to 151$)$ & $<0 \cdot 0001$ \\
\hline
\end{tabular}

doctors can see at a glance what has been omitted. The colour coding of the preprinted sheets makes them easier to find in a thick set of notes.

An important element of our preprinted sheets are the spaces left for further detail. This design prevents doctors ringing words and phrases as a mechanical clerking exercise. We not only ask the junior doctors to record all the core clinical details but also emphasise that they must show judgement in the way that they record the appropriate additional details.

The benefits of the preprinted notes have been sustained over one year. Others reported that monthly audit of randomly selected notes led to a considerable improvement in medical inpatient notes over one year, ${ }^{1}$ which, however, was not sustained when monitored over three years. ${ }^{2}$ We are confident that the improvements observed in our admission notes will be sustained for the following reasons: continued use of preprinted notes, an introductory teaching session about the notes for all new junior doctors, and daily feedback to junior doctors on the morning ward round.

Preprinted assessment sheets have been used in specialist units in managing specific conditions such as asthma. ${ }^{3}$ We have found no published account of their use for general medical, surgical, or paediatric admissions. We are continuing to modify the sheets and have recently introduced integrated inpatient paediatric notes which incorporate them. (Integrated notes are shared among doctors, nurses, and staff from other disciplines. ${ }^{4}$ ) Preliminary studies of integrated notes have suggested considerable benefits of their use. ${ }^{4}$ We continue to monitor our inpatient notes to see if the integrated notes further improve the quality of inpatient paediatric care compared with the preprinted notes alone.

The preprinted assessment sheets improve the quality and probably reduce the length of admission clerking. Different departments would choose different core clinical details, but with suitable modifications preprinted admission sheets could be used widely.

1 Gabbay J, McNicol MC, Spiby J, Davies SC, Layton AJ. What did audit achieve? Lessons from preliminary evaluation of a year's medical audit. $B M \mathcal{F}^{1} 1990 ; 301$ : 526-9.

2 Gabbay J, Layton AJ. Evaluation of audit of medical inpatient records in a district general hospital. Quality in Health Care 1993;1:43-7.

3 Town I, Kwong T, Holst P, Beasley R. Use of a management plan for treating asthma in an emergency management plan for treating asthm

4 Williams JG, Roberts R, Rigby MJ. Integrated patient records: another move towards quality for patients? Quality in Health Care 1993;2:73-4. 
Children's Department

Assessment Form

North Middlesex Hospital

Well this morning. Woke up from lunchtime sleep at 13.30 feeling hot. Sitting on settee when eyes became glazed and rolled up. All 4 limbs began to shake. Lasted for less than 2 minutes. Afterwards, floppy and drowsy until arrived in casualty at 14.00 . No previous episode.

Drugs taken in recent hours or days

Activity in last hours

Food in last hours

Fluid in last hours
Nil

Normal Definitely reduced Very little

Normal Definitely reduced Very little

Normal Definitely reduced Very little

\section{Previous medical history}

Birth weight

Pregnancy or perinatal problems Immunisations up to date

Previous serious illnesses

\section{Development}

Latest motor skill

Latest language skill

School progress

Development

\section{Family history}

Other relevant family history

\section{Social history}

Comfortable home

Other comments
$7 \mathrm{lbs} 4 \mathrm{oz}$

No or detail:

Yes or detail:

No or detail:

UTI - age 8/12. Investigations "normal"

Sits unsupported Crawls Cruises Walks Runs Babbles Single words Two word combinations

Short sentences Conversation

Preschool No problems or detail:

Normal Abnormal If abnormal then detail: 


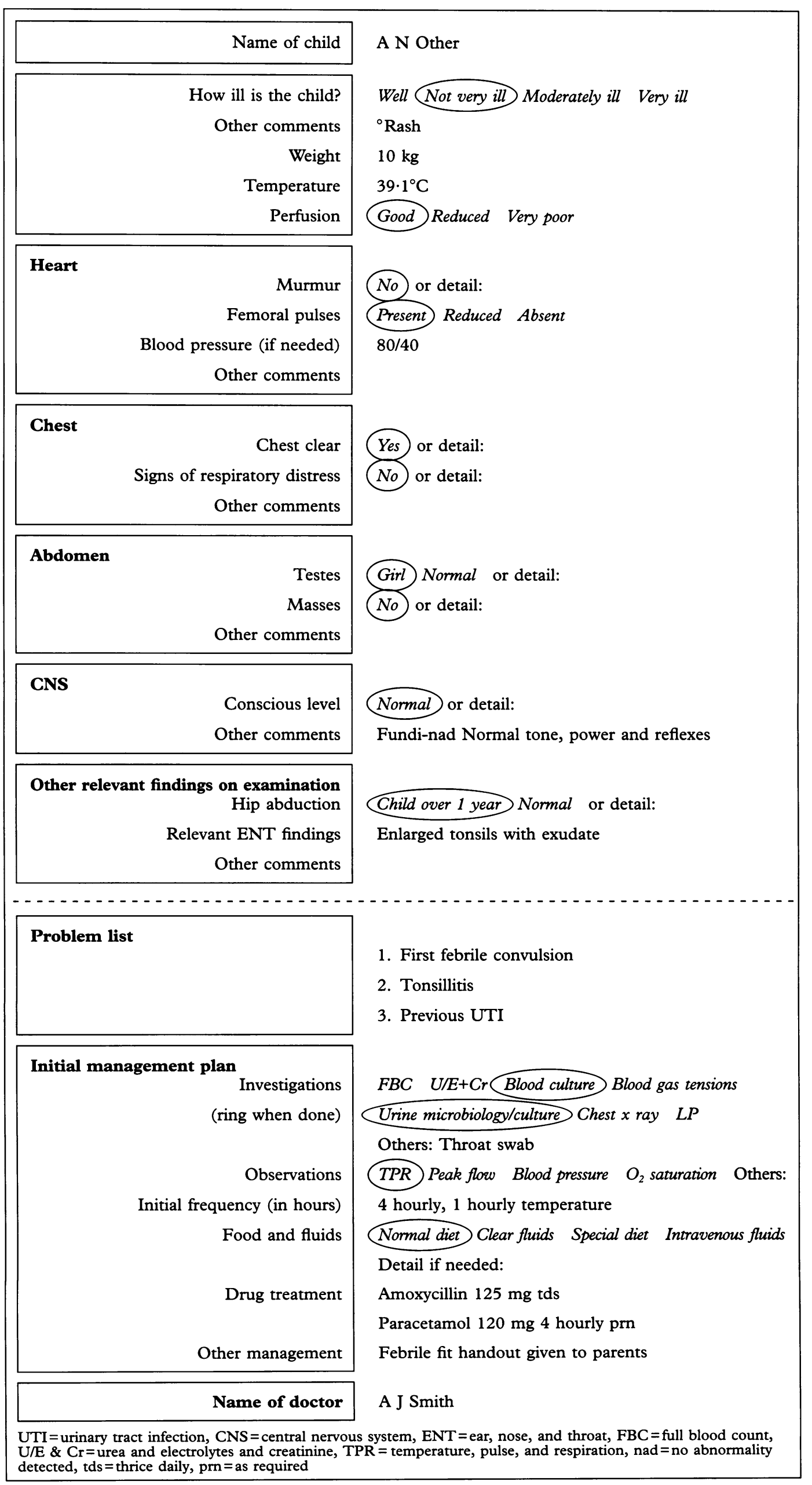

